\title{
Christine Desmoulins, Bernard Zehrfuss
}

Gollion/Paris, Infolio/Éditions du Patrimoine, 2008

\section{Xavier Dousson}

\section{(2) OpenEdition}

Journals

Édition électronique

URL : http://journals.openedition.org/crau/325

DOI : $10.4000 /$ crau.325

ISSN : 2547-5746

Éditeur

Éditions du patrimoine

Édition imprimée

Date de publication : 1 décembre 2009

Pagination : 211-215

ISBN : 978-2-85822-944-4

ISSN : 1296-4077

Référence électronique

Xavier Dousson, "Christine Desmoulins, Bernard Zehrfuss », Les Cahiers de la recherche architecturale et urbaine [En ligne], 24/25 | 2009, mis en ligne le 01 septembre 2017, consulté le 24 septembre 2020.

URL : http://journals.openedition.org/crau/325 ; DOI : https://doi.org/10.4000/crau.325

Ce document a été généré automatiquement le 24 septembre 2020.

Cahiers de la recherche architecturale et urbaine 


\section{Christine Desmoulins, Bernard Zehrfuss}

Gollion/Paris, Infolio/Éditions du Patrimoine, 2008

Xavier Dousson

\section{RÉFÉRENCE}

Christine Desmoulins, Bernard Zehrfuss, Gollion/Paris, Infolio/Éditions du Patrimoine, coll. «Carnets d'architectes », 2008. vol. 2, 192 pages, $20 €$.

1 Deuxième volume d'une toute jeune collection de monographies d'architectes, l'ouvrage de Christine Desmoulins marque à bien des égards (par son montage institutionnel, par son sujet éminent, par son positionnement critique) autant une forme d'aboutissement qu'un prometteur commencement.

\section{«Carnets d'architectes », une collection attendue}

2 Depuis plus d'une vingtaine d'années, la mobilisation croissante des historiens de l'architecture sur des sujets de recherche liés aux architectures de la Reconstruction et de la Croissance a permis de réévaluer progressivement la production de ces années d'après-guerre au premier choc pétrolier. Contextualisée, cette production vilipendée par la vague critique des années 1970 - même si elle est encore largement stigmatisée dans les médias généralistes - est regardée de nouveau avec moins de partialité et de mépris.

3 Cette réévaluation critique a été, en France et de manière décisive, rendue partiellement possible par l'action du Centre d'archives d'architecture du $\mathrm{xx}^{\mathrm{e}}$ siècle, situé à Paris et créé par Maurice Culot dans le cadre de l'Institut français d'architecture au début des années 1980. Après un peu plus d'un quart de siècle de fonctionnement, cet outil remarquable peut s'enorgueillir de conserver des centaines de fonds 
d'architectes qui forment de fait une véritable collection, vaste et impressionnante, de documents souvent inédits. Dès lors, et depuis de nombreuses années déjà, se pose la question de la diffusion et de la valorisation de ce patrimoine par une action d'ampleur qui permettrait de dépasser la seule publication de jalons, souvent magnifiques et substantiels, mais trop rares et exceptionnels ${ }^{1}$.

4 Après plusieurs projets avortés ${ }^{2}$, celui qui est porté conjointement par la toute jeune maison d'édition suisse Infolio fondée en 1999 et les vénérables, institutionnelles et françaises Éditions du Patrimoine semble en passe de réaliser cette ambition. En effet, la publication de l'ouvrage de Christine Desmoulins, le deuxième volume de cette nouvelle collection, mais le premier co-édité par les deux maisons d'édition, est largement "alimenté » par le fonds Zehrfuss conservé au Centre d'archives. Il devrait être bientôt suivi d'autres volumes co-édités ${ }^{3}$ qui viendront se placer entre ceux directement publiés par Infolio, comme celui qui est consacré à la figure de Buckminster Fuller, le premier de la série ${ }^{4}$. La collection comble ainsi une attente. Elle le fait remarquablement en consacrant ses deux premiers volumes à des figures importantes et incontestables de l'architecture contemporaine, figures pour lesquelles il n'existait jusqu'alors aucun ouvrage monographique en langue française ${ }^{5}$.

\section{L'ambition d'un projet éditorial}

5 Le petit format de l'ouvrage, à peine plus grand qu'un A5 $(21 \times 17 \mathrm{~cm})$, et son prix relativement modique (20 euros) ne doivent pas masquer l'ambition qui a présidé à la naissance de cette collection. Celle-ci se place en effet volontairement à égale distance des volumineuses monographies d'architectes, souvent scientifiquement ambitieuses, et des collections plus populaires dont de nombreux éditeurs européens et anglosaxons se sont fait une spécialité ${ }^{6}$. Les premières, généralement coûteuses, semblent réservées à un public limité, tandis que les secondes, souvent peu convaincantes en matière de contenu et d'appareil scientifique, ressassent d'ordinaire les mêmes sujets.

6 La collection explore ainsi un positionnement original. Plus raffinée et scientifique que les secondes, moins élitiste et coûteuse que les premières, elle essaie de combiner l'exigence d'un texte critique et l'attractivité d'une collection économique et séduisante sur le plan iconographique. Ne refusant pas les sujets ardus (après tout, aucun éditeur ne s'était lancé jusqu'à présent dans la publication de monographies sur Fuller ou Zehrfuss en français!), elle ambitionne en près de 150 pages d'offrir pour chaque sujet un document de référence, une sorte de vade-mecum de «ce qu'il faut connaitre » de l'architecte abordé(e) et de son œuvre.

7 Pour cela, elle adopte une structuration de ses volumes là aussi relativement inédite. Un portfolio ouvre l'ouvrage. Ramassé en peu de pages, résumant le parcours de l'architecte en quelques visuels et légendes, il précède un texte critique et théorique plus substantiel. La vocation de cet essai est de dessiner un portrait du concepteur et, surtout, de mettre en évidence l'originalité de sa démarche architecturale et de ses choix, tant doctrinaux ou théoriques que pratiques ou historiques. Après cette introduction qui fixe les cadres d'une interprétation, quelques projets représentatifs (six dans les deux premiers volumes) sont détaillés individuellement par un texte de présentation et une large sélection de visuels. Une liste complète (exhaustive ?) des projets et réalisations, accompagnée d'une bibliographie consistante et des éléments biographiques principaux, clôt l'ouvrage. D'une certaine manière, cette liste invite à 
poursuivre les investigations, elle ouvre naturellement des pistes de recherches ultérieures.

Enfin, dernière ambition de cette collection, la volonté de ses animateurs de lui offrir des livraisons régulières. Après un démarrage timide de deux volumes en 2008, passé presque inaperçu, plusieurs autres sont publiés en 2009 et courant $2010^{7}$, de manière à établir durablement la présence de ce projet dans le paysage éditorial.

\section{Une identité visuelle originale}

9 Le titre de la collection, "Carnets d'architectes », et la forme générale de l'ouvrage suggèrent bien qu'il s'agit d'autre chose que d'un simple essai sur une personnalité de l'architecture et sa production. Texte critique et iconographie sont, si ce n'est quantitativement à égalité, du moins à parité. Cette importance des documents visuels, parfaitement justifiée et bienvenue, est par ailleurs renforcée par un travail soigné en matière de présentation (composition, choix typographiques, graphisme). Celui-ci incite à aborder en quelques mots les questions de formalisation de l'ouvrage et souligne l'apport substantiel du graphiste, Sylvain Enguehard ${ }^{8}$, dont on peut écrire, même si son nom n'apparaît qu'en tout petit à l'intérieur de l'ouvrage, qu'il en est indéniablement l'un des auteurs, tant son apport est lisible, original et, sur quelques sujets, interroge.

Parmi les nombreux points positifs de cette collection, le choix du format apparaît comme une décision essentielle. Ni trop petit, condamnant les images à n'être que des timbres-poste, ni trop grand, obligeant à des compositions savantes pour combiner plusieurs reproductions, il engage à limiter le nombre d'illustrations par page, permettant ainsi de les révéler pour ce qu'elles sont. Pour l'essentiel, elles sont ainsi reproduites pleine page, accentuant leur vitalité et leur lisibilité. La qualité d'impression, la présentation en couleurs des documents et la distinction franche des textes et des illustrations renforcent encore cette présence et cette indépendance de l'iconographie. De toute évidence, celle-ci ne fait pas qu'illustrer le texte mais possède son autonomie. Elle forme la matière d'une partie de l'exigence de la collection illustrée par son titre, « Carnets d'architectes ».

11 Si l'effet collection - encore difficile à mesurer - ne fonctionne pas à plein, plusieurs choix graphiques interrogent le lecteur, généralement habitué pour ce type d'ouvrages à une présentation plus sobre et convenue. La typographie inhabituelle, les doubles pages de présentation des chapitres avec leurs images au floutage savant, les grands àplats récurrents bordeaux, la couverture occupée par un titre hiéroglyphique (bizarrement redoublé en haut à droite) et le marquage des légendes semblent des partis pris plutôt étonnants pour une collection qui ambitionne de durer. Cette recherche d'une identité formelle singulière qui, il faut le souligner, ne s'oppose jamais au contenu, apparait tout à la fois comme une manière efficace de se démarquer des autres publications courantes de ce type ${ }^{9}$, souvent bien moins imaginatives sur ces points, de souligner un positionnement éditorial singulier et de "dépoussiérer ", voire de renouveler, ce genre d'exercice. Pourtant, elle porte en elle le risque de dater l'ouvrage, donc toute la collection. Risque manifestement assumé par ses concepteurs dont on ne peut que souligner, une fois de plus, la volonté de renouvellement d'un genre quelque peu éculé. 
12 Deux points mineurs apparaissent cependant comme des maladresses un peu agaçantes. Le premier tient au légendage des images du portfolio introductif: leur repérage se fait au moyen de chiffres placés au strict milieu des documents, visibles comme le nez au milieu de la figure. Non seulement ces chiffres dégradent les documents originaux qu'ils renseignent et n'apportent rien, en matière de lisibilité, par rapport à des solutions plus classiques, mais ils donnent visuellement une place prépondérante à un appareillage qui ne devrait rester qu'une aide discrète à la compréhension. Le deuxième point tient au rognage systématique des images des chapitres ultérieurs pour y installer (ou non) des légendes. Si ce rognage apparaît comme une solution malicieuse pour toutes les fois où il permet aux documents de conserver d'amples dimensions, il se révèle comme une sorte de gimmick obsessionnel un peu incompréhensible lorsqu'il retaille inutilement des documents pour lesquels il n'y a pas légendes ${ }^{10}$ ou des surfaces libres limitrophes. Ce sentiment est encore renforcé lorsque le rognage masque des pans importants des documents visuels, au risque d'en perturber la lecture ${ }^{11}$. Ces pratiques de légendage, pas toujours convaincantes, mériteraient peut-être d'être réadaptées car elles gâchent une partie du travail graphique, par ailleurs subtil et d'une grande cohérence.

\section{La redécouverte ambiguë d'un architecte moderne}

13 À la mort de Bernard Zehrfuss (1911-1996), le spécialiste d'architecture du Monde appelait de ses vœux un travail historique sur l'architecte et concluait sa notice nécrologique par ces mots : "Tous ses bâtiments n'ont pas été d'égale réussite, mais toutes ses réalisations, par leur aspect grandiose (le CNIT, l'Unesco), par leurs hésitations urbaines (le Haut du Lièvre), et ses chefs-d'œuvre (le Musée de Lyon) retracent avec une rare fidélité l'aventure et les aléas du mouvement moderne via le style international. En retracer l'histoire serait faire œuvre pédagogique dans un pays comme la France, qui a eu quelque raison de vouloir oublier les années noires de son architecture dans les décennies qui ont suivi la guerre ${ }^{12}$.»

14 Cette invitation a été suivie scrupuleusement par Christine Desmoulins, auteur d'une longue recherche sur l'architecte sanctionnée par l'obtention d'une thèse ${ }^{13}$ et, par ailleurs, déjà auteur de plusieurs publications architecturales ${ }^{14}$. L'ouvrage vient donc combler une attente autant qu'il valorise un long travail de recherche, mais il le fait d'une manière quelque peu ambiguë.

15 Exact contemporain de Gillet, Albert, Dubuisson, Boileau et Henri-Labourdette, Le Couteur ou encore de Pouillon ou Wogenscky, Bernard Zehrfuss est le produit étonnant de la rencontre entre l'enseignement des Beaux-Arts, où il se forme entre 1928 et 1939 dans l'atelier d'Emmanuel Pontremoli, la modernité corbuséenne qu'il découvre tardivement pendant la guerre et la pratique des architectures vernaculaires tunisiennes. Au moment où il entame véritablement sa carrière d'architecte, tout auréolé de son grand prix de Rome, il est sensiblement confronté aux mêmes problématiques que ses illustres confrères. Or, celles-ci tiennent essentiellement aux questions posées par la Reconstruction (en Tunisie) puis les années de Croissance. Elles concernent principalement la construction en masse de logements dans des quartiers, voire des villes, qu'il faut souvent construire de toutes pièces. Sur ces sujets, Bernard Zehrfuss est de tous les combats (pour l'industrialisation, pour l'emploi de formes et de typologies modernes, pour l'obsession solaire, etc.) et de tous les concours importants 
du MRU (Villeneuve-Saint-Georges en 1949, Cité Rotterdam à Strasbourg en 1951). Très souvent associé à Jean Sebag pour la réalisation de logements, il construit à Alger, Nanterre, Bagnolet, Boulogne, Nancy, Paris, Saint-Étienne, Clichy-sous-BoisMontfermeil, etc. Il participe, sous la houlette de Marcel Lods, aux premières expériences, fondatrices, de construction de logements véritablement de masse ${ }^{15}$. Il est l'un des premiers à employer le chemin de grue, il pousse la rationalisation de la conception et de la construction de logements à des niveaux inhabituels et marque ainsi de son empreinte le paysage de nombreuses villes françaises.

Sur cet immense travail, sur cet engagement, l'ouvrage est, comme du reste la thèse de l'auteur, relativement discret, se contentant de deux paragraphes et de quelques vignettes. Christine Desmoulins préfère présenter en détail des œuvres exceptionnelles et incontestables de Zehrfuss, des productions valorisantes et séduisantes sur le plan plastique, ayant fait l'objet d'un travail poussé et plus personnel sur la spatialité, des réalisations souvent publiées, jusqu'à aujourd'hui encore ${ }^{16}$. Or ce choix, volontaire ${ }^{17}$ et très peu explicité, apparaît comme curieux, pour plusieurs raisons.

La première est qu'il semble accréditer, implicitement, le préjugé populaire de la médiocrité de ces architectures de logements de masse, comme s'il n'y avait rien à en sauver, comme si la dimension plastique des projets devait presque être l'unique critère de sélection des œuvres. Pourtant ces productions de logements ont été, en leur temps, des moments importants de l'histoire de l'architecture française, comme du reste se le rappelait l'architecte peu de temps avant sa mort, soulignant l'importance des recherches techniques qui avaient été menées à l'époque : « Il fallait jouer le jeu, parce que le but était de faire progresser des techniques de construction. L'enjeu était de répondre à des critères d'économie et de vitesse. Il fallait suivre les essais de préfabrication, même ceux qui étaient peu réussis comme le procédé Camus. On sait que cela a été un casse-gueule terrible, mais on a joué le jeu parce que cela faisait progresser les techniques du bâtiment, les techniques d'entreprise. [...] Il est très certain qu'il y a eu beaucoup de heurts à ce moment-là, mais je trouve que ce qui a été fait à l'époque était malgré tout assez courageux. Cela indiquait une tendance qui visait à améliorer la construction ${ }^{18}$. "

Des inventions et expérimentations techniques, de la mise au point de solutions rationnelles ou économiques, de l'exploration des ressources offertes par l'industrialisation du bâtiment, du travail souvent ingénieux de mise au point des plans de logements, de l'importance des conditions de production, de la contestation de ces architectures de masse et de l'autocritique ultérieure de l'architecte, Christine Desmoulins ne dit presque rien. Pourtant, lorsqu'on songe à l'icône de cette politique de construction de masse que fut et reste l'opération du Haut du Lièvre à Nancy (Actuellement en cours de rénovation par Alexandre Chemetoff et ses associés), cette discrétion ne cesse d'interroger. Elle renferme potentiellement le risque de présenter l'architecte comme une sorte de Docteur Jekyll et Mister Hyde de l'architecture, créateur inventif et talentueux lorsqu'il s'attaque à des commandes prestigieuses, constructeur médiocre, puisque son travail n'est pas ou peu présenté dans l'ouvrage, lorsqu'il réalise des logements. Cette survalorisation du créateur artiste au détriment du constructeur efficace, historiquement contestable, perpétue également, malgré les réévaluations critiques et le foisonnement des travaux des historiens contemporains de la discipline (qui la présentent depuis longtemps comme une pratique complexe et non uniquement artistique), une vision quelque peu Beaux-Arts de l'histoire de 
l'architecture, en contradiction possible avec le positionnement professionnel même de Zehrfuss. Au fond, elle ne donne pas entièrement à comprendre la singularité du positionnement de l'architecte.

19 Enfin, au moment où de vastes projets de recomposition des Grands Ensembles voient le jour, incités par l'empilement des politiques publiques, en particulier celle de l'ANRU, interroger plus sensiblement la production de masse de l'architecte aurait peut-être pu servir à une plus juste prise en compte de l'intérêt de cette production, souvent négligée par les promoteurs de ces opérations de rénovation, qu'ils soient architectes ou maîtres d'ouvrage.

\section{Une publication essentielle. Des images saisissantes}

Malgré cette prévention et ces interrogations, il faut savoir gré à Christine Desmoulins d'avoir présenté avec beaucoup de soin la production de l'architecte, restituant avec justesse le rôle de plusieurs collaborateurs de l'édification, qu'ils soient concepteurs ou maîtres d'ouvrage. Les projets présentés sont tous éminemment intéressants, des jalons importants d'une carrière et d'une époque. De fait, cet ouvrage est indispensable pour qui veut connaitre l'architecte, tant il regorge d'informations et ouvre de questionnements. Pour qui veut enrichir sa vision de la période, il est tout aussi essentiel. Ses qualités en font probablement, et pour longtemps, un document de référence. Elles invitent l'auteur et/ou d'autres chercheurs à poursuivre leurs recherches sur Zehrfuss, elles appellent à la publication scientifique des mémoires de l'architecte.

21 Et si la volonté de l'auteur de ne révéler que les «beautés indiscutables» de l'architecture de Zehrfuss interroge, elle a le mérite de dévoiler certains projets méconnus (l'imprimerie Mame et le gratte-ciel de la Défense) et de renseigner sur l'état oublié des édifices à leur livraison. À ce titre, les images du CNIT en version originale, libéré de la gangue du parvis qui l'enserre et de son remplissage actuel, le montrent dans toute la clarté de son dessin primitif, à la fois si franc et limpide. Elles expriment mieux que des mots l'immense gâchis de ses remaniements successifs dont la construction de la tour Phare (Morphosis-Thom Mayne, architectes), qui devrait littéralement en absorber la façade nord-est, apparaît comme l'ultime avatar.

\section{NOTES}

1. Semblant contredire ces propos, le Centre d'archives édite depuis plusieurs années un bulletin, Colonnes, dont la mission est de faire le lien entre chercheurs et institutions sur l'ensemble des questions posées par la conservation des archives d'architectes. Adressé d'abord aux professionnels, ce périodique ne bénéficie cependant que d'une diffusion confidentielle. Par ailleurs, des nombreux ouvrages édités s'appuyant largement et principalement sur les fonds d'archives du Centre, certains semblent vouloir former collection, en particulier ceux publiés par les éditions Norma (Maurice Culot, David Peycéré et Gilles Ragot, Les frères Perret, l'œuvre complète, 
2000 ; Susan Day, Jean-Charles Moreux, 2001; Jean-Baptiste Minnaert, Henri Sauvage, 2002 et Maurice Culot et Anne Lambrichs, Albert Lapradre, 2007). Pourtant, la rareté de ces publications ne peut prétendre répondre à la question posée, celle d'une valorisation large et volontaire de l'ensemble du patrimoine du Centre d'archives.

2. Notamment ceux développés lorsque Jean-Louis Cohen était à la tête de la Cité de l'architecture, où ceux soutenus directement par la direction du Centre d'archives (David Peycéré) et ses salariés (Éric Furlan).

3. Ces ouvrages doivent beaucoup à la détermination de Simon Texier, porteur du projet depuis plusieurs années et codirecteur de la collection avec Paolo Amaldi, et au soutien financier et éditorial de la DAPA (Élisabeth Henry).

4. Federico Neder, Buckminster Fuller, coll. «Carnets d'architectes », volume I, Infolio, Gollion, 2008.

5. L'ouvrage, capital, construit à partir de fragments d'interviews donnés par Fuller à son gendre, Buckminster Fuller : an Autobiographical Monologue Scenario, publié en anglais en 1980, a été traduit en français en 2004. Néanmoins, il ne constitue pas véritablement une monographie au sens habituel du terme. Voir Buckminster Fuller, Scénario pour une autobiographie, entretiens réunis par Robert Snyder, trad. Didier Semin, Paris, éditions Images Modernes, 2004.

6. Collections chez Taschen (Basic Architecture series), Motta Architettura (Minimum), Gustavo Gili (Works and Projects), Electa, Studio Paperback, Skira (bibliothèque d'architecture), etc.

7. La collection devrait s'enrichir des titres suivants : Henri Sauvage, Lúcio Costa, Georges-Henri Pingusson, Auguste Perret, Émile Aillaud, Archigram, Aldo Rossi, Beaudoin et Lods, Louis Arretche, etc.

8. http://www.sylvainenguehard.com. S. Enguehard est, entre autres, le graphiste d'ouvrages remarqués d'architecture pour le pavillon de l'Arsenal, la Cité de l'architecture et du patrimoine, le Centre Georges Pompidou ou encore des architectes contemporains.

9. Cette démarcation n'apparaît pas explicitement comme un objectif publicitaire ou simplement commercial. En témoigne cette couverture, plus impressionniste et moins directe que celle de la plupart des ouvrages monographiques édités.

10. Par exemple pages 83 et 143 .

11. Par exemple pages $85,87,104,163$.

12. Frédéric Edelmann, «Bernard Zehrfuss, un grand architecte français », rubrique Disparition, Le Monde, vendredi 5 juillet 1996, p. 11.

13. Les jalons de cette recherche sont essentiellement : Bernard Zehrfuss (1911-1996) : itinéraire d'un architecte, mémoire de DEA d'histoire socioculturelle, université de Versailles-Saint-Quentin-enYvelines, 2001 (dir. François Loyer) et Bernard Zehrfuss, un architecture français (1911-1996). Une figure des Trente Glorieuses, thèse de doctorat en histoire de l'architecture, université de VersaillesSaint-Quentin-en-Yvelines, 2 juillet 2008 (dir. François Loyer).

14. Plusieurs publications de $\mathrm{Ch}$. Desmoulins se rapportent déjà au genre de la monographie d'architecte. Voir Adrien Fainsilber et associés 1986-2002, Paris, Éditions Alternatives, 2003 et Joseph Belmont, parcours atypique d'un architecte, Paris, PC Éditions, 2006. Autres ouvrages : aux Éditions Le Moniteur, 25 Maisons individuelles, Paris, 2002 ; 25 Musées, 2005 ; 25 Maisons en bord de mer, 2007 ; (dir.), Le collège des Bernardins - histoire d'une reconversion, Paris, Éditions Alternatives, 2009.

15. Voir en particulier Marcel Lods, «à propos de l'industrialisation du bâtiment, 5000 logements dans la région parisienne », Techniques \& Architecture, no 5, 1959, pp. 92-95.

16. Toutes (ou presque) les monographies de Nervi et Breuer présentent le palais de l'Unesco (à l'exception bien sûr des édifices ultérieurs construits par le seul Zehrfuss). Le CNIT fait l'objet d'un long chapitre dans l'ouvrage de Bernard Marrey sur Esquillan, Nicolas Esquillan, un ingénieur d'entreprise, Paris, éditions Picard, 1992. L'usine Renault de Flins est largement détaillée par Corinne Bélier dans Les bâtisseurs de la modernité, 1940-1975, AMO-Le Moniteur, Paris, 2000, pp. 46-53. 
17. La collection offre «normalement » la possibilité de détailler cinq projets architecturaux. L'auteur en présente six, dont certains sur des sites communs (le CNIT et le projet de gratte-ciel à La Défense) ou sur des programmes proches (les installations industrielles de l'imprimerie Mame et de l'usine Renault).

18. Jean Balladur - Bernard Zehrfuss, conférence du 7 février 1991 animée par Bruno Vayssière, coll. « Architectes repères, repères d'architectures : 1950-1975 », les mini PA, no 20, Paris, Éditions du pavillon de l'Arsenal, 1998,

\section{AUTEURS}

\section{XAVIER DOUSSON}

Architecte, enseignant à l'Énsa de Paris-Malaquais, doctorant à l'université de Paris 1-PanthéonSorbonne, membre du LACTH, Énsap de Lille. 